\title{
Intensive short-term dynamic psychotherapy provided by novice psychotherapists: effects on symptomatology and psychological structure in patients with anxiety disorders
}

\author{
Diego Rocco, ${ }^{1}$ Vincenzo Calvo, ${ }^{2}$ Vito Agrosì, ${ }^{3}$ Francesca Bergami, ${ }^{3}$ Luce Maria Busetto, ${ }^{3}$ Silvia Marin, ${ }^{3}$ \\ Giovanna Pezzetta, ${ }^{3}$ Luca Rossi, ${ }^{3}$ Lorenzo Zuccotti, ${ }^{3}$ Allan Abbass ${ }^{4}$
}

${ }^{1}$ Department of Developmental and Social Psychology, University of Padova, Padova, Italy; ${ }^{2}$ Department of Philosophy, Sociology, Pedagogy and Applied Psychology, University of Padova, Padova, Italy; ${ }^{3}$ C.I.S.S.P.A.T. Centro Italiano Studio Sviluppo Psicoterapie A breve Termine, Padova, Italy; ${ }^{4}$ Centre for Emotions and Health, Dalhousie University, Canada

\begin{abstract}
This study examines the effectiveness of psychotherapy provided by novice therapists, in an attempt to clarify the controversial relationship between treatment effectiveness and therapist experience. To achieve this, we examined the short- and long-term effectiveness of intensive short-term dynamic psychotherapy (ISTDP) in the treatment of patients with the Diagnostic and Statistical Manual of Mental Disorder, IV edition - Text Revision anxiety disorders, as provided by novice psychology trainees. Twenty-two patients with anxiety disorders were provided ISTDP. Patients improved significantly on all outcome indices, including the global assessment of functioning, the symptom checklist and the inventory of interpersonal problems, at the end of the treatment and at 6 and 12 month follow-up. In addition to these results, there was marked structural personality change as evidenced by ratings on the Shedler Westen assessment procedure (SWAP-200), at the same assessment moments; the SWAP-200 psychological health index score showed a meaningful increase in adaptive psychological resources and capacities, while the mean number of personality diagnoses decreased from the beginning to the end of therapy, and all patients maintaining their gains in 6-12 month follow-up. We conclude that ISTDP provided by novice psychotherapists is efficacious in bringing broad and in-depth change to pathology that can perpetuate anxiety disorders and other psychiatric conditions.
\end{abstract}

Key words: Intensive short-term dynamic psychotherapy; outcome; anxiety disorders; trainees.

Correspondence: Diego Rocco, Department of Developmental and Social Psychology, University of Padova, Via Belzoni 84, 35121 Padova, Italy.

Tel.: +39.0498.278498. E-mail: diego.rocco@unipd.it

Citation: Rocco, D., Calvo, V., Agrosì, V., Bergami, F., Busetto, L.M., Marin, S., Pezzetta, G., Rossi, L., Zuccotti, L., \& Abbass, A. (2021). Intensive short-term dynamic psychotherapy provided by novice psychotherapists: effects on symptomatology and psychological structure in patients with anxiety disorders. Research in Psychotherapy: Psychopathology, Process and Outcome, 24(1), 413. doi: 10.4081 /ripppo. 2021.503

Received for publication: 1 October 2020.

Revision received: 10 November 2020

Accepted for publication: 27 November 2020.

This work is licensed under a Creative Commons Attribution NonCommercial 4.0 License (CC BY-NC 4.0).

${ }^{\circ}$ Copyright: the Author(s), 2021

Licensee PAGEPress, Italy

Research in Psychotherapy:

Psychopathology, Process and Outcome 2021; 24:4-13

doi:10.4081/ripppo.2021.503

\section{Intensive short-term dynamic psychotherapy provided by novice psychotherapists: effects on symptomatology and psychological structure in patients with anxiety disorders}

One of the main goals in psychotherapy research is to demonstrate the efficacy of a psychotherapeutic treatment model (Levy, Ablon, \& Kächele, 2012). The establishment how much a specific treatment can improve a patient's psychological well-being is of great theoretical, economical and ethical importance.

In order to establish the effectiveness of psychotherapy, it is necessary to go beyond self-report measurements - for instance self-reported symptom reduction, improvement of the patient's capacity to relate to others in their daily life, changes in dysfunctional behaviors - and objectively assess outcomes (Wampold \& Imel, 2015). Objective measures overcome the limits of the subjective clinical evaluation that are routinely used to evaluate clinical effectiveness and extend the degree to which psychotherapy's effectiveness can be confirmed.

From the methodological point of view, randomized 
controlled trials (RCT) (Shadish, Cook, \& Campbell, 2002) control treatment variables enabling a reliable analysis of the efficacy of a psychotherapy treatment. Repeated RCTs also allow a systematic accumulation of data on which treatments work and in which context (Gelo et al., 2010).

However, even if RCTs are recognized for their reliability, many scholars (Persons \& Silberschatz, 1998; Westen, Novotny, \& Thompson-Brenner, 2004) underline the problems connected with the strict limitations that RCT impose. RCTs can create a distance between the results obtained by the analysis in the laboratory and the results as obtained in the daily normal clinical practices. This may especially be the case with young psychotherapists where the distance between lab and normal practice may great for various reasons including the quality of the received training (Rocco, Gennaro, Filugelli, Squarcina, \& Antonelli, 2019), the impact of the therapist characteristics (Lingiardi, Muzi, Tanzilli, $\&$ Carone, 2018) and the ability to recognize and manage countertransference (Rocco, De Bei, Negri, \& Filipponi, 2021). In the attempt to understand the role these factors play in determining the intervention's effectiveness, scholars tried to compare the outcomes of psychotherapy provided by expert therapists to the outcomes of psychotherapy provided by less expert therapists. The results of these meta-analyses were no univocal: some of them found no correlation between years of experience and outcomes (Shapiro \& Shapiro, 1982; Smith, Glass, \& Miller, 1980), while one did find such a correlation (Stein \& Lambert, 1995).

For all these reasons, and especially in the case of young psychotherapists, it is important to integrate the results obtained RCTs, that constitute a fundamental basis for the evaluation of a specific treatment efficacy, with those obtained in a naturalistic scenario: such study provides information about a treatment's effectiveness, generalizability, feasibility and cost-effectiveness (Comer \& Kendall, 2013).

Naturalistic studies (Shadish et al., 2002) are founded on the quasi-experimental research design (Leichsenring, 2004; Shadish et al., 2002), where the patients are not selected on the basis of researchers' criteria, but as they are found in clinical practice. Despite this methodological difference, studies show that naturalistic studies do not overestimate effects compared to RCTs studies (Benson, 2000; Shadish et al., 2002).

Whether we examine RCT or naturalistic data, some minimal criteria must be present in order to obtain reliable data. First, a specific intervention and technique must be identified with an adherence system. Second, evaluation of not only a symptom improved evaluation but also personality structure evaluation should be included. Finally, maintenance of any accrued gains should be evaluated in follow up (Comer \& Kendall, 2004; Gelo et al., 2010).

Using these 3 parameters, we evaluated a specialized treatment model intensive short-term dynamic psychotherapy (ISTDP) (Abbass et al., 2014; Abbass, 2015; Davanloo, 2000), whose efficacy has been widely studied for a broad range of common mental disorders demonstrating large treatment effect sizes that continue or even increase in longterm follow-up (Abbass, Kisely, Rasic, \& Katzman, 2013; Lilliengren, Johansson, Lindqvist, Mechler, \& Andersson, 2016). Recent studies of ISTDP show that it is effective with depression, panic disorder, somatic symptom disorders and personality disorders (Abbass, Town, \& Driessen, 2012, 2013; Lilliengren, Cooper, Town, Kisely, \& Abbass, 2020). A recent study found ISTDP effective and cost-effective with generalized anxiety disorder and 2 recent randomized controlled trials found ISTDP to be efficacious for social anxiety disorder (Rahmani, Abbass, Hemmati, Ghaffari, \& Rezaei Mirghaed, 2020a; Abbass, Hemmati, Mirghaed, \& Ghaffari, 2020b).

We sought to examine whether ISTDP is transferable to novice psychotherapists, whether it is effective in their hands and whether it maintains its effectiveness over time. We have chosen to analyze the effectiveness of this specific intervention model because of its effectiveness and the availability of a group of independent young professionals to participate to the research. Despite of all these features, ISTDP is also, according to Flegenheimer (1982), the intervention model most difficult to learn among the other short-term dynamic intervention models, because is characterized by a high degree of therapist activity in response to clinical phenomena and treatment inclusion criteria that force the therapist to continually verify that the patient is able to tolerate the treatment.

After the pioneering work of Davanloo $(1980,1990)$ the ISTDP treatment model, in addition to the traditional way of learning psychotherapy founded on the oral or written transmission of the knowledge (Freud, 1905, 1909a, 1909b), uses a more technological training modality. Through the use of video technology in didactic teaching, case practice and supervision, it is possible not only to listen and read the therapy reports, but also to see and listen to the psychotherapy live through video review (Abbass, 2004; Abbass et al., 2013b). The advantages of this learning method are clear: the psychotherapy setting is open and other therapists can directly learn a way of doing psychotherapy live. Video review offers more in-depth case examination including the non-verbal communications of both therapist and patient (Abbass, 2004).

In previous research, psychiatrists in training were able to facilitate symptom reduction on the Brief Symptom Inventory (BSI; Derogatis, \& Spencer, 1982) and interpersonal problem gains with the Inventory of Interpersonal Problems (IIP; Horowitz, Straub, \& Kordy, 2000) while treating a mixed patient sample using ISTDP (Abbass, 2004, Abbass et al., 2013a). This second study also found that resident-treated cases had greater healthcare use reduction if the treating resident had more hours of supervision. There are currently no studies exclusively 
examining ISTDP outcomes from novice psychotherapists or psychologists in training.

One of the main goals of this article is to demonstrate, using objective research data within a naturalistic research design, that novice psychotherapists can provide effective treatment bringing lasting symptom and personality structural changes.

To pursue this goal, we evaluated treatments of patients with anxiety disorders with or without comorbidities. We choose anxiety disorders because they are one of the most prevalent mental disorders encountered in both primary mental health services and in private practice (Fonagy, Roth, \& Higgitt, 2005). In fact, at an international level, the lifetime prevalence of anxiety disorders is $16.6 \%$ (Somers, Goldner, Waraich, \& Hsu, 2006).

This work is part of a wider research project on ISTDP developed without financial support from external funding agencies.

\section{Objectives}

The first objective of this study is to examine the effectiveness of the ISTDP (Abbass et al., 2012; Davanloo, 2000 ) in outpatients with anxiety disorders. In addition to symptom reduction we will examine deeper changes in underlying psychological and personality processes that give rise to symptoms. From the psychodynamic perspective, addressing such underlying drivers is considered critical in order to prevent relapse and to reduce ongoing morbidity. If an intrapsychic or structural change has occurred can we say that the patients' problems have been resolved at some depth.

A second objective following is to verify whether ISTDP is effective when applied by novice psychotherapists who obtained the title of psychotherapist less than two years prior.

This objective offers an implicit evaluation of the degree to which the ISTDP approach is transmissible while maintaining the effectiveness already recognized in the literature (Abbass et al., 2012; Abbass et al., 2013b; Rhamani et al., 2020b).

Finally, we evaluate whether the results are stable over time, by means of two follow up clinical interviews.

\section{Methods}

\section{Enrollment}

The patients in this study had Diagnostic and Statistical Manual of Mental Disorder, IV edition - Text Revision (DSM-IV TR) anxiety disorders treated by the clinicians in their own private practices. The sample was composed of Italian people $(\mathrm{n}=22)$, derived from self-referred or from clinician-referred patients. They were 14 females and 8 males from age 18 to 51, with a mean age of 33.5 [standard deviation $(\mathrm{SD})=9.88$ ]. Twelve were married or in a long-term relationship and ten were single. Seventeen were employed (13 were employees, 3 were self-employed and 1 was an executive), three were students, and one was unemployed. Patients were included in the research if they had an anxiety disorder diagnosed by the clinician using DSM-IV TR (see Results section). Based on the high degree of comorbidity among the different kind of anxiety disorders and between anxiety disorders and other disorders (for example personality disorders, substance disorders etc.; Andrews, Slade, \& Issakidis, 2002), comorbidity was not an exclusion criterion. Presence of psychotic disorder was an exclusion criterion.

\section{Therapists}

Treatment courses were provided by seven psychotherapists, four women and three men, mean age 35 years (range 30 to 40 years). All held a degree in Psychology and had completed training to the professional level of psychotherapist on average 1.5 years prior (range 1-2 years). These therapists were in training in ISTDP provided by certified ISTDP trainers. At the time of the research they had not fully completed their 400 hours of ISTDP training.

\section{Independent raters}

The coders of GAF and SWAP-200, apart the clinicians themselves, were other of the therapists in a rotation and the research coordinator who was a male, experienced psychotherapist, supervisor, and researcher in psychotherapy. The coders were blind regarding the clients' therapeutic results.

\section{Treatment and adherence}

ISTDP is a psychotherapy intervention model created by Habib Davanloo $(1990,2000)$. By means of a detailed video analysis of clinical materials, from several hundred treated cases, Davanloo defined an effective psychotherapy intervention characterized by short duration and effectiveness with a broad range of patients. In a face -to-face setting and with an active, engaged therapist, the patient is enabled to systematically override chronic defenses to get in touch with their previously repressed emotions which are considered to be at the root of their symptoms and character problems.

The flexibility of the approach, the rapid diagnosis conducted through the identification of both unconscious anxiety pathways ${ }^{1}$ and the major defenses utilized by the patient, together with the changes in technique of recent years, allow the application of ISTDP to almost $86 \%$ of outpatients referred to psychiatrists (Abbass, 2002).

The metapsychological basis of ISTDP, derived from detailed case study, links to both psychoanalytic psy-

i.e. the ways in which the anxiety show its presence that, in ISTDP, is classified in: i) Striated (voluntary) muscle unconscious anxiety; ii) smooth muscle unconscious anxiety; iii) cognitive-perceptual disruption; iv) conversion (Abbass, 2015). 
chotherapy and Bowbly's attachment model (1982). It considers psychopathology to be the effect of emotional trauma as well as interruptions of bonds with attachment figures. These events provoke such strong and complex emotions that the person is forced to block or avoid them, especially when they occur in early phases of development. Subsequent events can mobilize emotions similar to the ones of the past, thus, activating unconscious anxiety and at the same time defenses that propel the behavioral and symptom disorders patients experience.

One of the main goal of ISTDP, is to help patients to overcome their difficulties in a short time. Thus, active interventions are used to allow the patient to experience their own genuine, previously unexpressed, pathogenic feelings as soon as possible within the treatment process. These processes involve the therapist helping the patient to directly overcome the emotional blocking mechanisms. Complex feelings that are mobilized in the session help the patient to understand, at an experiential level, how they usually block what they feel. In this way therapist and patient examine previously repressed complex feelings in a moment to moment process. Once the anxiety and defenses are overcome, the therapeutic forces of the unconscious therapeutic alliance become activated (Abbass, 2015; Davanloo, 2000).

The breakthrough, referred to as unlocking is the moment that Davanloo has identified when the unconscious complex feelings become conscious and bring linkages and images of past conflicted emotions (Davanloo, 1980, 1990). Davanloo called this psychic force bringing the memories and linkages, the unconscious therapeutic alliance. Often one unlocking alone leads to symptom reduction with a single session (Abbass, Joffres, \& Ogrodniczuk, 2008, 2009; Abbass, Town, Ogrodniczuk, Joffres, \& Lilliengren, 2017). Usually more such breakthroughs are necessary in order to obtain more stable changes. After the unlocking of repressed emotions, the therapist, by means of specific techniques, helps the patient to cognitively consolidate the insights through detailed recapitulation.

Thus, ISTDP is both intensive, since the therapeutic process is accelerated due to therapeutic pressure on the emotional experience and the intensification of transference resistances, and experiential, since it permits a direct experience of the feelings involved.

As a psychodynamic framework of both verbal and non-verbal patients communication, ISTDP uses the triangle of the person (T-C-P; Transference (therapist), Current, Past) and the triangle of conflict (F-A-D; feelings, anxiety, defenses) that Malan (1963) used with the aim of allowing the therapist to orientate himself in his interactions with the patient. The triangles allow the description of the intrapsychic dynamic in which the patient defends himself from the anxiety generated by conflicted impulses or emotions in the current relationship, in the relationship with the therapist as well as with important people from the past.

The psychotherapy process is articulated in different phases which Davanloo termed the central dynamic sequence: i) inquiry: exploring the patient's difficulties; ii) pressure: leading to resistance in the form of a series of defenses; iii) challenge: identification, clarification and challenge to the defenses; iv) transference resistance: clarification and challenge to the transference resistance; v) breakthrough: intrapsychic crisis and high rise in the complex transference feelings; first unlocking of the unconscious; vi) systematic analysis of the transference; vii) further inquiry exploring the developmental history; viii) phase of direct access to the unconscious.

These phases don't always appear in this sequence, and often it is necessary to repeat some of these before obtaining the breakthrough to the unconscious conflicting emotions. The major result of all of this focused therapeutic work is that the time needed for psychodynamic treatment is greatly reduced.

In this research the treatment courses were an average duration of 23.95 hours (range 7-53 sessions). These were provided over an average 9.68 months (range 3 to 11). Each session lasted 90 minutes (in some case up to 120 minutes), with a weekly or biweekly frequency.

To ensure adherence to the ISTDP model in this study, all therapists received video-recording based supervision on their cases (on average 2.55 hours each; range 1-10 hours), conducted by a psychotherapist supervisor certified by the International Experiential Dynamic Therapy Association (IEDTA; www.iedta.net). Adherence was examined by reviewing session videos to identify the use of techniques from the central dynamic sequence.

\section{Measures}

After establishing adherence to the model, we organized outcome measurement by applying the following criteria. First, we used self-administered and therapist-administered atheoretical instruments. To do this we chose instruments capable of detecting both superficial (symptom) and deeper (personality structure) aspects of change. Finally, we verified the maintenance of any accrued gains in follow (Comer \& Kendall, 2004; Gelo et al., 2010).

The Global Assessment of Functioning (GAF; Hall, 1995), measures global functioning and is a tool derived from the Luborsky's Health-Sickness Scale (HSRS; Luborksy, 1962). The GAF detects general psychopathology aspects and is present on axis 5 of the DSM-IV TR. The scale includes values from 1 to 100 divided in ten sectors and each of them has ten points (from 1 to 10; from 11 to 20 and so on until the sector from 91 to 100 ). For example the first sector, from 1 to 10 , indicates an extremely low functioning characterized by 'Persistent danger of severely hurting self or others (e.g., recurrent violence) or persistent inability to maintain minimal personal hygiene or serious suicidal act with clear expectation of death', and the last sector, from 91 to 100 , indicates 'No symptoms. Superior functioning in a wide range of activities, life's problems 
never seem to get out of hand, is sought out by others because of his or her many positive qualities'.

The Symptom Check List (SCL-90 R; Derogatis, 1983; Prunas, Sarno, Preti, Madeddu, \& Perugini, 2012; Sarno, Preti, Prunas, \& Madeddu, 2011), is a self-report checklist composed of 90 items that contribute to a symptom profile of nine dimensions. Each item is evaluated on a five-point Likert scale ( $0=$ not at all; $4=$ extremely) that expresses how much the item describes the patient's experience during the last week. The nine dimensions are somatization, obsessivecompulsive, interpersonal sensitivity, depression, anxiety, hostility, phobic anxiety, paranoid ideation and psychoticism. Each dimension is significant if the value obtained is equal or greater than 1.5. This tool is atheoretical and can be used in different time frames of treatment, allowing the assessment of whether symptom change has occurred. Given the specific aim of this research, we chose to use the global severity index (GSI) and the anxiety (ANX) subscale.

The inventory of interpersonal problems (IIP-32; Horowitz et al., 2000; Lo Coco et al., 2018), is a self-report instrument for assessing interpersonal relationship problems. The patient evaluates 32 items characterizing his or her interpersonal relationships on a five-point Likert scale $(0=$ not at all; $4=$ extremely). This tool is atheoretical and allows the description of interpersonal behavior on two main dimensions: i) Cold to Self-sacrificing, varying on a continuum from hostile behavior to friendly behavior, and ii) Domineering to Nonassertive, varying from submitted behavior to controlling or dominating behavior.

Eight scales derive from these two dimensions: Domineering - being too aggressive, Vindictive - being suspicious and distrustful, Cold - lacking affection and sympathy, Socially inhibited - being socially anxious and shy, Nonassertive - failing to be assertive, Overly accommodating - being too trusting and permissive, Self-sacrificing - being too eager to please others, Intrusive seeking attention inappropriately. The transformed $t$ values can vary from 36 to 99 , and a value over 70 is considered significant indicating some relationship problems on that specific dimension.

We assessed personality function using the SWAP-200 - Shedler Westen Assessment Procedure (Shedler \& Westen, 2007). The SWAP-200 is an objective measure completed by a trained clinical observer. This instrument is based on the Q-sort method, a psychometric method designed to maximize reliability and minimize error variance. The instrument has 200 personality-descriptive items or statements, each of which may describe a given person well, somewhat, or not at all. SWAP-200 items are written in jargon-free language and provide a standard vocabulary for clinical case description that can be used by clinicians of all theoretical orientations. The assessor sorts or ranks the statements into eight categories, from most descriptive (scored 7) to not descriptive or irrelevant (scored 0). Thus, the SWAP-200 instrument yields scores from 0 to 7 for 200 personality-descriptive variables.
When the assessor completes the scoring procedure, software-based scoring algorithms combine and weight the item scores to compute diagnostic scales (Shedler, 2009). The SWAP-200 software generates three score profiles: the first provides a DSM-IV TR/DSM5 personality disorder diagnoses. The second score profile provides diagnostic scores for an alternative set of personality syndromes derived through empirical research ('Q-factors'). These empirically derived personality syndromes were developed to more accurately map the personality syndromes seen in real-world clinical practice (Westen \& Shedler, 1999; Westen, Shedler, Bradley, \& DeFife, 2012). A third SWAP-200 score profile (not reported here) provides dimensional trait scores (Shedler \& Westen, 2004). Additionally, the SWAP-200 includes a Psychological Health Index that measures adaptive personality resources and capacities. All diagnostic scale scores are reported as $\mathrm{T}$-scores (mean $=50, \mathrm{SD}=10$ ) based on norms established in a national clinical sample.

The SWAP-200 provides scale scores assessing the following eleven empirically-identified personality syndromes: dysphoric, antisocial, schizoid-schizotypal, paranoid, obsessional, histrionic, narcissistic, avoidant, high functioning depressive, borderline (emotionally dysregulated), dependent-victimized, and, hostile-externalizing personality (Shedler, 2009; Westen \& Shedler, 1999). The SWAP-200 scale scores permit the detection of personality changes.

Although SWAP-200 assesses personality syndromes dimensionally, cut-points can be applied to make categorical diagnoses where desired to facilitate clinical communication. Scale scores of $\mathrm{T}>60$ warrant a categorical personality disorder diagnosis (the patient has the disorder) and scores of $\mathrm{T}>55$ warrant a diagnosis of subthreshold traits or features (the patient has significant features of the disorder).

\section{Administration of outcome measures}

Patients provided written consent to participate in research and to be video recorded. In lieu of the naturalistic and quasi experimental nature of this study with inherent low risk, the project was not reviewed by an ethics committee.

All the psychotherapists involved in the research project received specific training on the use and administration of the instruments presented by an expert researcher.

The GAF outcomes were completed by each psychotherapist at the end of first consultation session, after the last psychotherapy session, at the end of the first (six month) and of the last (one year) follow up clinical interview. The four GAF were independently completed by two members of the research team (included the research coordinator) based on viewing the video records of these sessions. The interclass correlation coefficient (ICC) (Shrout \& Fleiss, 1979) of the assessment of the independent raters was $0.73,0.81,0.74$ and 0.73 in these 4 
time points. These scores were compared, and if they were in the same sector of the GAF, the mean data were used; if some differences were present then the therapist was included in a discussion with the colleagues until reaching a consensus. Both the IIP-32 and the SCL-90R were given to the patient to complete before the first consultation session, after the last psychotherapy session and before the two follow up clinical interviews.

With the SWAP-200, therapists evaluated the first two sessions and the last two sessions of the treatments, the first (six month) and of the last (one year) follow up clinical interview. Usually three to five sessions are needed to apply the SWAP methodology, but since the duration of an ISTDP session was twice as long as traditional psychotherapy, we evaluated fewer sessions.

Since the SWAP-200 assessments are usually completed by the therapists, and in our case the therapists are included in the research program, the research team, to avoid conflicts of interest, made the following choice: after the therapist produced his own SWAP-200 profile, other members of the research team (at least two, included the research coordinator), did the same based on viewing the same video recorded sessions. For each of these four time points they produced a distinct SWAP assessment. During their assessment the researchers were blind to which sessions (early, late or follow-ups) they were rating. The ICC (Shrout \& Fleiss, 1979) of the assessment of the independent raters was, in the four time points $0.88,0.75$, 0.78 and 0.71 . The so obtained scores were compared, and if they were equal regarding the dimensions (both PD and $Q$ factors) over and under the cut-offs, the data were used; if some differences were present (i.e. the same dimensions were not over, or under, the same cut-off), then the therapist was included in a discussion with the colleagues until reaching a consensus.

\section{Data analysis}

Descriptive analyses and repeated measures analysis of variance (ANOVA) were conducted to compare pre, post, and follow-ups results for GAF, SCL-90R's scales,
IIP-32 data and the SWAP-200's PHI. The GreenhouseGeisser correction was applied when the assumption of sphericity could not be assumed. Pairwise post hoc comparisons with a Bonferroni adjustment were carried out on pre, post, and follow-up outcomes (Table 1). For evaluating the deeper changes in underlying psychological and personality process, we did a quantitative analysis of the SWAP-200 profiles.

In order to evaluate the clinically significance of SCL90R anxiety scale changes at the individual patient level, we adopted the standard psychometric procedure suggested by Speer (1992). With this procedure, to address concerns of pretreatment score regression to the mean, each of the pretest scores used in this stage of data analysis was adjusted to attenuate any regression effects. Reliable change index scores (RCI) (Jacobson \& Truax, 1991) were then calculated for each variable (using the adjusted pretest scores); an RCI score exceeding 1.96 suggests that the test score change was psychometrically reliable, reflecting real change. Moreover, each of the three posttreatment test scores then was examined to determine whether it fell below the cutoff score for a functional distribution, within 2 SDs of the normative mean. If both of these criteria were met (i.e., reliable change and moved within $2 \mathrm{SD}$ of the normative mean), individuals were considered to have achieved clinically significant change. RCIs were also examined to determine whether any individuals reliably deteriorated over the course of treatment. All calculations of clinical significance for the SCL-ANX subscale used the normative mean (0.30, SD 0.37) and test-retest reliability $(0.85)$.

\section{Results}

\section{Sample diagnosis}

The therapist and the raters who applied the SWAP methodology also assessed the two initial sessions with the DSM-IV TR (American Psychiatric Association, 2000) Axis I criteria, and they used a consensus procedure to establish Axis I diagnosis. Their initial agreement was

Table 1. Analysis of pre-post and follow-up data.

\begin{tabular}{|c|c|c|c|c|c|c|}
\hline Measure & $\begin{array}{c}\text { Mean (SD) } \\
\text { T1 }\end{array}$ & $\begin{array}{c}\text { Mean (SD) } \\
\text { T2 }\end{array}$ & $\begin{array}{c}\text { Mean (SD) } \\
\text { T3 }\end{array}$ & $\begin{array}{c}\text { Mean (SD) } \\
\text { T4 }\end{array}$ & $\begin{array}{c}F \text { value } \\
\left(d_{\text {ftime }}, d_{\text {ferror }}\right)\end{array}$ & Partial $\eta^{2}$ \\
\hline GAF & $55.28(4.21)$ & $80.11(5.57)^{*}$ & $80.56(4.89)^{*}$ & $78.89(4.73)^{*}$ & $213.76 * *(1.956,33.249)$ & 0.93 \\
\hline SCL-ANX & $2.03(.68)$ & $0.42(0.31)^{*}$ & $0.37(0.27)^{*}$ & $0.30(0.18)^{*}$ & $90.12 * *(1.260,21.415)$ & 0.84 \\
\hline SCL-GSI & $1.51(.43)$ & $0.35(0.20)^{*}$ & $0.29(0.21)^{*}$ & $0.28(0.17)^{*}$ & $102.64 * *(1.343,22.824)$ & 0.86 \\
\hline IIP-32 & $59.17(8.42)$ & $49.33(5.43)^{*}$ & $46.33(5.38)^{*}$ & $45.78(5.39)^{*}$ & $27.35 * *(1.746,29.682)$ & 0.62 \\
\hline SWAP-PHI & $54.24(7.52)$ & $64.69(4.01)^{*}$ & $66.01(4.43)^{*}$ & $64.79(4.28)^{*}$ & $48.96 * *(1.395,23.714)$ & 0.74 \\
\hline
\end{tabular}

SD, standard deviation; T1, beginning of therapy; T2, end of therapy; T3, 6 months follow-up; T4, 12 months follow-up; GAF, global assessment of functioning; SCL-ANX, anxiety scale of the SCL-90-R; SCL-GSI, global severity index of the SCL-90R; IIP-32, inventory of interpersonal problems; SWAP-PHI, psychological health index of the SWAP-200. *P $<0.05$; there was a statistically significant decrease from T1 to T2, from T1 to T3, and from T1 to T4 on all measures, but not from T2 to T3 and T3 to T4 (Post hoc tests using the Bonferroni correction); ** $<0.001$; one-way repeated measures ANOVAs with a Greenhouse-Geisser correction indicated statistically significant differences on outcome measures between T1, T2, T3 and T4. 
91\%. Diagnoses were: panic disorder $(\mathrm{n}=6)$, social phobia $(n=4)$, generalized anxiety disorder $(n=9)$, agoraphobia $(\mathrm{n}=1)$, specific phobia $(\mathrm{n}=1)$ and unspecified anxiety disorder $(\mathrm{n}=1)$. According to the PD profile in the SWAP score, two patients showed avoidant personality disorder, five showed dependent personality disorder, one had obsessive-compulsive personality disorder and fourteen had no Axes II diagnosis ${ }^{2}$.

\section{Therapist effects}

We estimated the variance in outcomes attributable to therapist random effects by evaluating the degree to which outcomes varied from zero for any outcome measure. ICCs for ANX and GSI scales of SCL-90R, GAF and for 3 scales of IIP-32 were null and nonsignificant ( $\mathrm{P}>0.05)$.

\section{Evaluation of treatment changes}

Repeated measures ANOVAs were conducted to determine whether there were statistically significant differences on outcome measures GAF, SCL-90R ANX, SCL-90R GSI, IIP-32 and SWAP-PHI between pre-therapy, post-therapy, 6-month follow-up and 12 month follow-up (see Table 1). Data for all patients were available for pre-therapy and post-therapy, while data the four time periods were available for 18 out of the 22 patients.

On the GAF, statistically significant changes in scores over time were detected $\left(F_{(1.956,33.249)}=213.76, \mathrm{P}<0.001\right.$, partial $\eta^{2}=0.93$ ). Post hoc analysis indicated a statistically significant increase from pre-therapy to post-therapy $\left(M_{\text {diff }}=-24.835,95 \%\right.$ confidence interval (CI) [-29.49, 20.17], P 0.001), from pre-therapy to 6-month follow-up $\left(M_{\text {diff }}=-25.28,95 \%\right.$ CI $[-29.37,-21.18]$, P 0.001$)$, and from pre-therapy to 12-month follow-up $\left(M_{\text {diff }}=-23.61\right.$, $95 \%$ CI $[-27.63,-19.60]$, P 0.001$)$, but not from posttherapy to 6-month and 12 month follow-up.

Scores on the SCL-90R ANX varied as a function of time $\left(F_{(1.260,21.415)}=90.12, \mathrm{P}<0.001\right.$, partial $\left.\eta^{2}=0.84\right)$. There were significant decreases in scores from pre-therapy to post-therapy $\left(M_{\text {diff }}=-1.611,95 \%\right.$ CI $[1.083,2.14], \mathrm{P}$

Patients with score in SWAP PD dimensions over the cut-off of 60 but with high functioning over the same cut-off, were not considered as patients with personality disorders.
0.001), from pre-therapy to 6-month follow-up $\left(M_{\text {diff }}=1.668,95 \%\right.$ CI $\left.[1.16,2.18], \mathrm{P} 0.001\right)$, and from pretherapy to 12 -month follow-up $\left(M_{\text {diff }}=1.732,95 \%\right.$ CI $[1.27,2.19], \mathrm{P} 0.001)$, but not from post-therapy to 6month follow-up and 12 month follow-up.

Also scores on the SCL-90R GSI varied as a function of time $\left(F_{(1.343,22.824)}=102.64, \mathrm{P}<0.001\right.$, partial $\left.\eta^{2}=0.86\right)$. There were significant decreases in scores from pre-therapy to post-therapy $\left(M_{\text {diff }}=1.158,95 \% \mathrm{CI}[0.82,1.50], \mathrm{P}\right.$ 0.001 ), from pre-therapy to 6-month follow-up $\left(M_{\text {diff }}=1.218,95 \% \mathrm{CI}[0.86,1.57], \mathrm{P} 0.001\right)$, and from pretherapy to 12 -month follow-up $\left(M_{\text {diff }}=1.229,95 \%\right.$ CI $[0.94,1.51], \mathrm{P} 0.001)$, but not from post-therapy to 6month follow-up and 12 month follow-up.

Changes in scores over time were also observed on the IIP-32 $\left(F_{(1.746,29.682)}=27.35, \mathrm{P}<0.001\right.$, partial $\left.\eta^{2}=0.62\right)$. Scores decreased significantly from pre-therapy to posttherapy $\left(M_{\text {diff }}=9.83,95 \%\right.$ CI $\left.[4.78,14.88], \mathrm{P} 0.001\right)$, from pre-therapy to 6-month follow-up $\left(M_{\text {diff }}=12.833,95 \%\right.$ CI $[6.76,18.91], \mathrm{P} 0.001)$, and from pre-therapy to 12-month follow-up $\left(M_{\text {diff }}=13.389,95 \%\right.$ CI $\left.[6.48,20.30], \mathrm{P} 0.001\right)$, but not from post-therapy to 6-month follow-up and 12 month follow-up. Specifically, the scores decreased significantly for all IIP scales apart the Vindictive one, that decreases but not in significant way.

Lastly, the score of the SWAP-200's Psychological Health Index showed a change over time $\left(F_{(1.395}\right.$, 23.714) $=48.96, \mathrm{P}<0.001$, partial $\left.\eta^{2}=0.74\right)$. Scores increased significantly from pre-therapy to post-therapy $\left(M_{\text {diff }}=-\right.$ $10.449,95 \%$ CI $[-15.01,-5.89]$, P 0.001$)$, from pre-therapy to 6 -month follow-up $\left(M_{\text {diff }}=-11.778,95 \% \mathrm{CI}\right.$ $[-16.26,-7.30], \mathrm{P} 0.001))$, and from pre-therapy to $12-$ month follow-up $\left(M_{\text {diff }}=-10.550,95 \%\right.$ CI $[-14.61,-6.48]$, P 0.001), but not from post-therapy to 6-month follow-up and 12 month follow-up.

\section{Clinically significant change in anxiety symptoms}

All (100\%) of patients had post treatment and follow up SCL-ANX scores in the normal, functional range (see Table 2). Reliable and clinically significant change in ANX scores was observed in $73 \%$ of patients at post therapy and $83 \%$ of patients in follow up. None of the patients deteriorated during psychotherapy on the SCL-ANX subscale. Moreover, we examined the distribution of SCL-

Table 2. Clinically significant change in anxiety symptoms.

\begin{tabular}{|c|c|c|c|}
\hline Criterion & SCL-ANX $(T 2 ; n=22)^{a}$ & SCL-ANX $(T 3 ; n=18)^{a}$ & SCL-ANX $(T 4 ; n=18)^{\mathrm{a}}$ \\
\hline $\mathrm{RCI}>1.96^{\mathrm{b}}$ & $16(73 \%)$ & $15(83 \%)$ & $15(83 \%)$ \\
\hline Functional distribution $^{\mathrm{c}}$ & $22(100 \%)$ & $18(100 \%)$ & $18(100 \%)$ \\
\hline Clinical significance $^{\mathrm{d}}$ & $16(73 \%)$ & $15(83 \%)$ & $15(83 \%)$ \\
\hline Deterioratione & $0(0 \%)$ & $0(0 \%)$ & $0(0 \%)$ \\
\hline
\end{tabular}

SCL-ANX, anxiety scale of the SCL-90-R; T2, end of therapy; T3, follow-up 6 months; T4, follow-up 1 year; RCI, reliable change index. ${ }^{a}$ Anxiety subscale of the symptom checklist; ${ }^{b}$ number of individuals who reliably improved after adjusting pretest scores for regression to the mean; 'number of individuals who fell within 2 standard deviations of the general population mean; dnumber of individuals who reliably improved and fell within 2 standard deviations of the general population mean; enumber of individuals who reliably deteriorated during treatment. 
90R ANX scale in relation to the criteria of Curran, West, and Finch (1996) for normality (i.e., skew, <2.0; kurtosis, $<7.0$ ) and found that our sample's ANX at Evaluation, ANX-Final, ANX at the follow ups, ANX-Raw Change, and ANX-RCI scores were all well within these limits (all variables skew $<0.75$ and kurtosis $<0.427$ ).

\section{Changes in underlying psychological and personality process}

Table 3 illustrates the average of the number of personality disorder diagnoses (SWAP-200 scale score of $\mathrm{T}>60)$ and diagnoses of subthreshold traits diagnoses (SWAP-200 scale score of T $>55$ ) for DSM-IV TR/DSM5 disorders assessed at the beginning and at the end of treatment, and at the follow ups. The mean number of personality diagnoses decreased from the beginning to the end of therapy and at the follow ups. This was true for both categorical personality disorder diagnoses (values of $\mathrm{T}>60$ ) and for diagnoses of subthreshold personality disorder traits (values of $\mathrm{T}>55$ ).

\section{Discussion}

Our first objective was to evaluate the effectiveness of ISTDP for anxiety disorders when provided by novice psychotherapists training in ISTDP with limited clinical experience. GAF values indicated a substantial increase in the global functioning of patients. Patients reported significant symptom improvement on the Anxiety subscale as well as GSI of the SCL-90. The treatments also facilitated interpersonal gains, as demonstrated by changes in the total score of IIP-32. These data suggest both that ISTDP is effective for anxiety disorders and that novice psychotherapists can effectively employ this method.

The mean SWAP-200 PHI score increased by approximately one standard deviation from beginning to end of treatment, from 54.24 to 64.69 , indicating a meaningful increase in adaptive psychological resources and capacities (ego strengths). Moreover, the treatment appears to have brought in depth changes in underlying psychological and personality processes that give rise to symptoms. Personality aspects that at the beginning of the therapies were dysfunctional (for instance dependency traits, represented in the SWAP-200 by item as 'Tends to be passive and unassertive' or 'Tends to be ingratiating or submissive') were substituted by more functional traits. These data suggest the changes due to ISTDP treatment seem to be at a deep level, working on intrapsychic conflicts and resultant personality structures that may manifest as anxiety disorders. Finally, these results were maintained in follow-up, suggesting that they are stable over time.

All these data show that beneficial effects of ISTDP when provided by experienced clinicians are also achievable by novice psychotherapists during training. This appears true not only for changes at level of symptom remission (Rahmani, et al., 2020a; 2020b), but also at the level of structural/personality change (Abbass et al., 2012; Abbass et al., 2013b; Lilliengren et al., 2020). Our findings provide information about role of limited clinical experience on clinical effectiveness and suggest that factors beyond clinical experience may contribute to outcomes. Indirectly, our results can be read as a validation of the ISTDP training model employing technology-based analysis of clinical intervention both during supervision and training (Abbass, 2004).

These results should be considered within the limits of this study's methodology. First the study had a relatively small sample suggesting a wider sample should be studied to assess the generalizability of the results. Second, patients studied had a medium/high level of adaptive personality resources, as measured by SWAP PHI, and therefore, may have been more likely to respond to treatment. Third, there was a relatively small pool of therapists, with an uneven distribution of the patients among them. Fourth outcomes were subjective in nature even though the SWAP was rated independently where possible. Finally, $20 \%$ of patients were not available for the follow up assessments. Because we lack a comparison group of more experienced therapists, we cannot conclude that these effects are equal, greater or less than those of more seasoned clinicians.

\section{Conclusions and future developments}

Converging data from multiple measures suggest that ISTDP in the hands of recently-trained psychologists is an effective treatment facilitating symptomatic, interpersonal and structural personality changes. The patients analyzed were not highly selected patients, apart from the anxiety symptoms reported to the therapist; hence, they represent typical patients that a therapist might face in private practice. This suggests that ISTDP may be widely applied to real world patients with anxiety disorders.

Future research in this ongoing project will involve a larger sample of anxiety disordered patients and the collec-

Table 3. Mean number of dimensions with a value over cut-off per patient at the beginning, the end and at follow-up assessments.

\begin{tabular}{lcccccccc}
\hline & \multicolumn{2}{c}{ T1 $(\mathbf{N}=\mathbf{2 2})$} & \multicolumn{2}{c}{ T2 $(\mathbf{N}=\mathbf{2 2})$} & \multicolumn{2}{c}{ T3 $(\mathbf{N}=\mathbf{1 8})$} & \multicolumn{2}{c}{ T4 $(\mathbf{N}=\mathbf{1 8})$} \\
\hline & $>60$ & $>55$ & $>60$ & $>55$ & $>60$ & $>55$ & $>60$ & $>55$ \\
\hline DSM personality disorders & 0.64 & 1.68 & 0.00 & 0.05 & 0 & 0.16 & 0.00 & 0.22 \\
\hline
\end{tabular}

DSM, Diagnostic and Statistical Manual; T1, beginning of therapy; T2, end of therapy; T3, follow-up 6 months; T4, follow-up 1 year. Values $>60=$ categorical diagnoses; values $>55=$ traits. 
tion of data from patients with other disorders. Another research path in development consists of detailed analysis of key processes and micro-processes that underly ISTDP treatment including the therapeutic alliance (Rocco, Gennaro, De Bei, Zanelli, \& Condino, 2013) and paraverbal aspects (Rocco, Mariani, \& Zanelli, 2013; Rocco, Gennaro, Salvatore, Stoycheva, \& Bucci, 2017; Rocco, et al., 2018).

\section{References}

Abbass, A. (2002). Office based research in intensive short-term dynamic psychotherapy (ISTDP): data from the first 6 years of practice. Bulletin of Short-term Dynamic Psychotherapy, $6(2), 5-13$

Abbass, A. (2004). Small group videotape training for psychotherapy skills development. Academic Psychiatry, 28(June), 151-155.

Abbass, A. (2015). Reaching through resistance: advanced psychotherapy techniques. Kansas City: Seven Leaves Press.

Abbass, A., Joffres, M. R., \& Ogrodniczuk, J. S. (2008). A naturalistic study of intensive short-term dynamic psychotherapy trial therapy. Brief Treat Crisis Interven. 8, 164-170.

Abbass, A., Joffres, M. R., \& Ogrodniczuk, J. S. (2009). Intensive short-term dynamic psychotherapy trial therapy: qualitative description and comparison to standard intake assessments. Ad Hoc Bulletin of STDP, 13(1), 6-14.

Abbass, A., Kisely, S., Rasic, D., \& Katzman, J. W. (2013a). Residency training in intensive short-term dynamic psychotherapy: Methods and cost-effectiveness. Psychiatric Annals, 43(11), 508-512.

Abbass, A. A., Kisely, S. R., Town, J. M., Leichsenring, F., Driessen, E., De Maat, S., \& Crowe, E. (2014). Short-term psychodynamic psychotherapies for common mental disorders. Cochrane Database of Systematic Reviews, 7, Article CD004687. http://dx.doi.org/10.1002/14651858.CD004687. pub4

Abbass, A., Town, J., \& Driessen, E. (2012). Intensive shortterm dynamic psychotherapy: a systematic review and metaanalysis of outcome research. Harvard Reviews of Psychiatry, Mar-Apr, 20(2), 97-108.

Abbass, A., Town, J. M., \& Driessen, E. (2013b). Intensive short-term dynamic psychotherapy: a review of the treatment method and empirical basis. Research in Psychotherapy: Psychopathology, Process and Outcome, 16(1), 6-15.

Abbass, A., Town, J., Ogrodniczuk, J., Joffres, M., \& Lilliengren, P. (2017). Intensive short-term dynamic psychotherapy trial therapy: Effectiveness and role of "unlocking the unconscious." Journal of Nervous and Mental Disease, 205(6), 453-457.

American Psychiatric Association. (2000). Diagnostic and statistical manual of mental disorders (4 $4^{\text {th }}$.ed., text rev.). Washington, DC: American Psychiatric Association.

Andrews G., Slade T., \& Issakidis C. (2002). Deconstructing current comorbidity: data from the Australian national survey of mental health and well-being. The British Journal of Psychiatry, 181, 306-14.

Bowbly, J. (1982). Attachment and loss: Vol 1. Attachment (2 $2^{\text {nd }}$ ed.). New York, NY: Basic Books.

Curran, P., West, S., \& Finch, J. (1996). The robustness of test statistics to nonnormality and specification error in confirmatory factor analysis. Psychological Methods, 1, 16-29.
Davanloo, H. (1980). Short-term dynamic psychotherapy. New York: Jason Aronson.

Davanloo, H. (1990). Unlocking the unconscious. Selected papers of Habib Davanloo. Toronto: John Wiley and Sons.

Davanloo, H. (2000). Intensive short-term dynamic psychotherapy. Selected papers of Habib Davanloo. Toronto: John Wiley and Sons.

Derogatis, L. R. (1983). Administration, scoring, and procedures manual for the recise version. Baltimore: Clinical Psychometric Research.

Derogatis, L. R., \& Spencer, P. M. (1982). The brief symptom inventory (BSI): administration, and procedures manual-I. Baltimore, MD: Clinical Psychometric Research.

Flegenheimer, W. V. (1977). Technique of brief psychotherapy. Northvale, NJ: Jason Aronson.

Fonagy, P., Roth, A., \& Higgitt, A. (2005). Psychodynamic psychotherapies: evidence-based practice and clinical wisdom. Bulletin of the Menninger Clinic, 69(1), 1-58.

Freud, S. (1905). Fragment of an analysis of a case of hysteria. S.E., 7, 7-122.

Freud, S. (1909a). Analysis of a phobia in a five-year-old boy. S.E., 10, 5-149.

Freud, S. (1909b). Notes upon a case of obsessional neurosis. S.E., 10, 155-318.

Hall, R. C. (1995). Global assessment of functioning. A modified scale. Psychosomatics, 36(3), 267-75.

Horowitz, L. M., Straub, B., \& Kordy, H. (2000). Inventory of interpersonal problems (IIP). Gottingen: Hogrefe.

Jacobson, N., \& Truax, P. (1991). Clinical significance: a statistical approach to defining meaningful change in psychotherapy research. Journal of Consulting and Clinical Psychology, 59, 12-19.

Levy, R. A., Ablon, J. S., \& Kächele, H. (2012). Psychodynamic psychotherapy research. evidence-based practice and practice-based evidence. London: Springer.

Lilliengren, P., Cooper, A., Town, J.M., Kisely, S., \& Abbass, A. (2020). Clinical- and cost-effectiveness of intensive shortterm dynamic psychotherapy for chronic pain in a tertiary psychotherapy service. Australasian Psychiatry, 28(4), 414-417.

Lilliengren, P., Johansson, R., Lindqvist, K., Mechler, J., \& Andersson, G. (2016). Efficacy of experiential dynamic therapy for psychiatric conditions: a meta-analysis of randomized controlled trials. Psychotherapy, 53, 90-104.

Lingiardi, V., Muzi, L., Tanzilli, A., \& Carone, N. (2018). Do therapists' subjective variables impact on psychodynamic psychotherapy outcomes? A systematic literature review. Clinical Psychology and Psychotherapy, 25(1), 85-101.

Lo Coco, G., Mannino, G., Salerno, L., Oieni, V., Di Fratello, C., Profita, G., \& Gullo, S. (2018). The Italian version of the inventory of interpersonal problems (IIP-32): psychometric properties and factor structure in clinical and non-clinical groups. Frontiers in Psychology, 9, 341.

Luborsky, L. (1962). Clinicians' judgements of mental health. A proposed scale. Archives of General Psychiatry, 7, 407-417.

Malan, J. (1963). A study of brief psychotherapy. London, Tavistock, New York: Reprint by Plenum Press.

Prunas, A., Sarno, I., Preti, E., Madeddu, F., \& Perugini, M. (2012). Psychometric properties of the Italian version of the SCL-90-R: a study on a large community sample. European Psychiatry 27(8), 591-597.

Rahmani, F., Abbass, A., Hemmati, A., Ghaffari, N., \& Rezaei Mirghaed, S. (2020a). Challenging the role of challenge in intensive short-term dynamic psychotherapy for social anx- 
iety disorder: A randomized controlled trial. Journal of Clinical Psychology, 76(12), 2123-2132.

Rahmani, F., Abbass, A., Hemmati, A., Mirghaed, S. R., \& Ghaffari, N. (2020b). The efficacy of intensive short-term dynamic psychotherapy for social anxiety disorder: randomized trial and substudy of emphasizing feeling versus defense work. The Journal of Nervous and Mental Disease, 208(3), 245-251.

Rocco D., De Bei, F., Negri, A., \& Filipponi, L. (2021). The relation between self-observed and other-observed countertransference and session outcome. Psychotherapy. Advance online publication, https://doi.org/10.1037/pst0000356.

Rocco, D., Gennaro, A., De Bei, F., Zanelli, D., \& Condino, V. (2013a). Development and characteristics of the therapeutic alliance in a short term dynamic psychotherapy: a descriptive case study. Research in Psychotherapy: Psychopathology, Process and Outcome, 16(1), 33-41.

Rocco, D., Gennaro A., Salvatore S., Stoycheva V., \& Bucci, W. (2017). Clinical mutual attunement and the development of therapeutic process: a preliminary study. Journal of Constructivist Psychology, 30(4), 371-387.

Rocco D., Gennaro, A., Filugelli, L., Squarcina, P., \& Antonelli, E. (2019). Key factors in psychotherapy training: an analysis of trainers', trainees' and psychotherapists' points of view. Research in Psychotherapy: Psychopathology, Process and Outcome, 22(3), 369-378. doi: 10.4081/ripppo.2019.415.

Rocco, D., Mariani, R., \& Zanelli, D. (2013b). The role of nonverbal interaction in a short term psychotherapy: preliminary analysis and assessment of paralinguistic aspects. Research in Psychotherapy: Psychopathology, Process and Outcome, 16(1), 54-64.

Rocco, D., Pastore, M., Gennaro, A., Salvatore, S., Cozzolino, M., \& Scorza, M. (2018). Beyond verbal behavior: an empirical analysis of speech rates in psychotherapy sessions. Frontiers in Psychology, 9, 978.

Sarno, I., Preti, E., Prunas, A., \& Madeddu, F. (2011). SCL-90$R$ symptom checklist-90-R Adattamento italiano. Firenze: Giunti, Organizzazioni Speciali.

Shadish, W. R., Cook, T. D., \& Campbell, D. T. (2002). Experimental and quasi-experimental designs for generalized causal inference. Boston: Houghton Mifflin Company.

Shapiro, D. A., \& Shapiro, D. (1982). Meta-analysis of comparative therapy outcome studies: A replication and refinement.
Psychological Bulletin, 92(3), 581-604. doi: 10.1037/00332909.92.3.581

Shedler, J. (2009). Guide to SWAP-200 interpretation. Denver, CO: SWAP assessment LLC. Retrieved from: www.SWAPassessment.org.

Shedler, J., \& Westen, D. (2004). Dimensions of personality pathology: an alternative to the five factor model. American Journal of Psychiatry, 161, 1743-1754.

Shedler, J., \& Westen, D. (2007). The Shedler-Westen assessment procedure (SWAP): making personality diagnosis clinically meaningful. Journal of Personality Assessment, 89(1), 41-55.

Shrout, P., \& Fleiss, J. (1979). Intraclass correlations: uses in assessing rater reliability. Psychological Bullettin, 86, 420-428.

Smith, M. L., Glass, G. V., \& Miller, T. I. (1980). The benefits of psychotherapy. Baltimore, MD: Johns Hopkins University Press.

Somers, J. M., Goldner, E. M., Waraich, P., \& Hsu, L. (2006). Prevalence and incidence studies of anxiety disorders: a systematic review of the literature. Canadian Journal of Psychiatry, 51(2), 100-13.

Speer, D. (1992). Clinically significant change: Jacobson and Truax (1991) revisited. Journal of Consulting and Clinical Psychology, 67, 894-904.

Stein, D. M., \& Lambert, M. J. (1995). Graduate training in psychotherapy: are therapy outcomes enhanced? Journal of Consulting and Clinical Psychology, 63(2), 182-196. doi: 10.1037/0022-006X.63.2.182

Wampold, B. E., \& Imel, Z. E. (2015). The great psychotherapy debate: the evidence for what makes psychotherapy work. Abingdon, England: Routledge.

Westen, D., \& Shedler, J. (1999). Revising and assessing Axis II, Part 2: Toward an empirically based and clinically useful classification of personality disorders. American Journal of Psychiatry, 156, 273-285.

Westen, D., Shedler, J., Bradley, B., \& DeFife, J. (2012). An empirically derived taxonomy for personality diagnosis: Bridging science and practice in conceptualizing personality. American Journal of Psychiatry, 169, 273-284.

Westen, D., Novotny, C. M., \& Thompson-Brenner, H. (2004). The Empirical Status of Empirically Supported Psychotherapies: Assumptions, Findings, and Reporting in Controlled Clinical Trials. Psychological Bulletin, 130(4), 631-663. https://doi.org/10.1037/0033-2909.130.4.631. 